\title{
Metastasis of human gastric adenocarcinoma partly depends on phosphoinositide-specific phospholipase $\gamma 1$ expression
}

\author{
Luhua Zhuang', Bingchang Zhang', Guoqing Zeng², Lianzhi Dai', Hongliu Qian', \\ Tianhui Hu${ }^{1}$, Gang Song ${ }^{1}$, Bing Zhang ${ }^{1}$, Chun Xia ${ }^{2}$ \\ ${ }^{1}$ Medical School, Xiamen University, Fujian, China \\ ${ }^{2}$ Zhongshan Hospital, Xiamen University, Fujian, China
}

\begin{abstract}
It is known that phosphoinositide-specific phospholipases $\gamma 1$ (PLC $\gamma 1)$ can trigger several signalling pathways to regulate cell proliferation, differentiation, and metastasis. However, whether this kinase is highly expressive and active in human gastric adenocarcinomas, and whether it can play an important role in the development of the cancer, have not yet been investigated. The aim of the study was to investigate the expression of PLC $\gamma 1$ in human gastric adenocarcinoma, while the question of whether PLC $\gamma 1$ can be activated through protein kinase $\mathrm{B}(\mathrm{Akt})$ signalling pathways to regulate cell migration was further explored using human gastric adenocarcinoma BGC-823 cell line. The expression of PLC $\gamma 1$ in human adenocarcinoma was detected using immunohistochemical staining. The BGC-823 cells were cultured and treated with inhibitors or transfected with plasmid construction. The cell migration of BGC-823 cells was measured with wound healing assay, cell migration assay, and the ruffling assay. The expression levels of PLC $\gamma 1$ and its related signal molecules in BGC-823 cells were assessed using Western blot analysis or gelatine zymography assay. PLC $\gamma 1$ was highly expressed in human gastric adenocarcinomas, especially in the region with lymph node metastasis. It was shown that migration of BGC-823 cells in vitro depends on PLC $\gamma 1$ activation. This activation is mediated through Akt, an upstream of PLC $\gamma 1$ that triggers the PLC $\gamma 1 /$ extracellular signal-regulated kinase (ERK)/matrix metalloproteinase (MMP) pathway in BGC-823 cells. PLC $\gamma 1$ activities play an important role in the metastasis of gastric adenocarcinoma, and may serve as a potential therapeutic target in this type of cancer. (Folia Histochemica et Cytobiologica 2014, Vol. 52, No. 3, 178-186)
\end{abstract}

Key words: human gastric adenocarcinoma; PLC $\gamma 1$ expression; BGC-823 cell line; cell migration; Akt; ERK; MMP2/9; tissue microarrays

\section{Introduction}

It has been reported that approximately $90 \%$ of mortality from cancers arises from the metastatic spread of primary tumors to distant sites, including gastric

Correspondence address: B. Zhang, Prof. Medical School, Xiamen University, Fujian, China tel.: +592-2880528; fax: +865922188421

e-mail: cristal66@xmu.edu.cn;

C. Xia, M.D., Zhongshan Hospital

Xiamen University, Fujian, China

tel.: +592-2993080; fax: +865925921461

e-mail: chunxia@xmu.edu.cn adenocarcinoma [1]. Metastasis usually includes a complex series of steps in which cancer cells leave the original tumor site and migrate to other parts of the body via the bloodstream, the lymphatic system, or by direct extension. Inhibition of any one metastatic step - such as migration or invasion - could prevent tumor dissemination $[1,2]$. Recent studies suggest targeted molecular therapy as a potential solution in cancer therapy, because it is more effective than current treatments and less harmful to normal cells [3]. The regulation of certain key signalling molecules has been identified as crucial in tumor metastasis; the molecules involved in cell migration may thus serve as molecular targets for the treatment of tumor metastasis $[4,5]$. 
Several lines of evidence indicate that phosphoinositide-specific phospholipase $\gamma$ (PLC $\gamma$ ) is one of key signalling molecules that regulates tumor metastasis [6-9]. PLC $\gamma$ has two isoforms, PLC $\gamma 1$ and PLC $\gamma 2[6,7]$. Activation of PLC $\gamma$ may occur in response to either integrin receptors or growth factor-dependent pathways - including epidermal growth factor (EGF) - and induces hydrolysis of phosphatidylinositol 4,5-bisphosphate (PtdIns $(4,5) \mathrm{P} 2)$ to form the second messengers diacylglycerol (DAG) and inositol 1,4,5-trisphosphate (IP3), which in turn activate a number of signalling pathways to regulate cell metabolism and cell metastasis [6-9]. For example, activated PLC $\gamma$ has been thought to play a critical role in both cytoskeletal changes and in the migration associated with the metastatic process in cancer cells [8]. Preventing the activation of the PLC $\gamma$ signalling pathway could limit the metastatic potential of breast and prostate cancers [9]. Both stable and inducible PLC $\gamma 1$ downregulation resulted in an almost complete inhibition of breast cancer-derived experimental lung metastasis formation [10]. Thus, PLC $\gamma$ plays an important role in some types of metastatic cancer. However, it is not clear whether the downregulation of PLC $\gamma 1$ may play a similar role in human gastric adenocarcinoma, compared to other types of cancers, and the possible effects of its inhibition have yet not been explored.

In addition to $\mathrm{PLC} \gamma$, protein kinase $\mathrm{B}$ (PKB/Akt), another well-known regulator of tumor metastasis, has been shown to modulate the same physiological process in some types of cancer [11, 12]. Both PLC $\gamma$ and Akt are known to be involved in the regulation of the growth and migration of MDA-MB-468 breast cancer and SW480 colon cancer cells [11]. The interaction between PLC $\gamma$ and Akt was found to regulate the G2/M transition in MDA-MB-231 breast cancer cells [12]. Therefore, exploring the relationship of PLC $\gamma$ and Akt in cancer metastasis will greatly assist in understanding the regulatory mechanisms of PLC $\gamma 1$ underpinning cell migration.

In this project, we aimed to study the expression level of PLC $\gamma 1$ in human gastric adenocarcinoma and normal gastric mucosa tissue, and to further investigate the molecular mechanisms of the PLC $\gamma 1$ activity associated with Akt that underpins cell migration in the human gastric adenocarcinoma cell line, BGC-823 . The results of this project are intended to provide additional evidence that PLC $\gamma$ may be a potential target for cancer treatment, and especially for the treatment of gastric adenocarcinoma.

\section{Material and methods}

Reagents and antibodies. Antibodies against PLC $\gamma 1$, p-PL-C 11 (Y783), p-PLC 1 (S1248), Akt, p-Akt (S473), Extracel- lular signal-regulated kinases (ERK), p-ERK (T202/Y204), Matrix metalloproteinase-9 (MMP9), MMP2, Human influenza haemagglutinin (HA), myc, and $\beta$-actin were purchased from Cell Signalling Technology, Inc. (Beverly, MA, USA) and Santa Cruz Biotechnology (Santa Cruz, Freemont, CA, USA). Inhibitors (U73122 and Triciribine) were obtained from Sigma-Aldrich in China (Shanghai, China). Other reagents were of the highest grade commercially available.

Tissue specimens. Five fresh specimens of normal gastric mucosa tissue and twenty specimens of T2, T3, N0-N2 gastric adenocarcinoma tissue were obtained from Zhongshan Hospital, Xiamen University and assessed by the Tumor Node Metastasis (TNM) staging system. The study protocol and design were approved by the Ethics Committee of Zhongshan Hospital, Xiamen University (ID No. 20060607). Each specimen underwent protein extraction and Western blot analysis. The tissue microarray, which includes 45 specimens of adenocarcinoma and 10 of normal gastric mucosa, was purchased from Biomax, Inc. (Rockville, MD, USA) for immunohistochemical staining. All our clinical studies were conducted according to the principles expressed in the Declaration of Helsinki.

Cell culture. The human gastric cancer cell line, BGC-823, was obtained from the Shanghai Institute of Cell Biology, Chinese Academy of Sciences, Shanghai, China, and was maintained in RPMI1640 medium supplemented with $10 \%$ foetal bovine serum (FBS), $100 \mathrm{U} / \mathrm{mL}$ penicillin, and $100 \mu \mathrm{g} / \mathrm{mL}$ streptomycin, at $37^{\circ} \mathrm{C}$ in a water-saturated atmosphere of $5 \% \mathrm{CO} 2$.

Plasmid construction and transfection. Rat PLC $\gamma 1$ cDNA was N-terminally tagged with the HA sequence and subcloned into pRK5-HA (pRK5-HA/PLC $\gamma 1$ ). The Y783 and S1248 mutations were obtained by PCR (PLC $\gamma 1$ Y783A, 5'CAACCCTGGTTTCGCTGTGGAGGCCAACCCTATGC (forward primer), 5'TTGGCCTCCACAGCGAAACCAGGGTTGCGGCC (reverse primer); PLC $\gamma 1$ S1248A, 5'CCGGGAAGGGGCCTTTGAAGCCAGATACCAGCAG (forward primer), 5' CTGGCTTCAAAGGCCCCTTCCCGGGCCCG (reverse primer). The ShPLC $\gamma 1$ vector (primer: 5'CCGGCCAGATCAGTAACCCTGAATTCTCGAGAATTCAGGGTTACTGATCTGTTTTTG3') was purchased from Gene Chem (Shanghai, China). The different expression vectors of PLC $\gamma 1$ and myc-Akt were transfected into BGC-823 cells using Lipofectamine 2000, according to the manufacturer's procedure (Invitrogen, Carlsbad, CA, USA). Briefly, BGC-823 cells were cultured in a $60-\mathrm{mm}$ dish for $20-24 \mathrm{~h}$ until they reached $60-70 \%$ confluence. The plasmid-containing buffer was then added and left for $36 \mathrm{~h}$, and was followed by the various experimental procedures in which the expression level of PLC $\gamma 1$ and Akt were detected with Western blot analysis, prior to the other experiments. 
Wound healing assay. The assay was conducted according to published protocol [13]. Briefly, a total of $2 \times 10^{6}$ cells were seeded onto two six-well plates and allowed to reach full confluence. Two centrelines were marked on the upside of each well along its horizontal axis, to designate the loci at which images would be acquired at each time point. Vertical linear scratches were introduced into the cell monolayers using a $10 \mu \mathrm{L}$ sterile pipette tip. Each well received 2 scratches. Culture plates were washed with PBS and incubated with PLC $\gamma$ inhibitor U73122, which was followed by EGF treatment in serum-free medium. Images at $40 \times$ magnification were acquired at $0,7,24$, and $48 \mathrm{~h}$ after scratching at each intersection of the scratch wound (vertical defect). For each time point, 4 measurements were taken per well in each of 3 wells, and the average of the horizontal width of the linear defect in pixels was calculated using the Image-Pro Plus 6.0 system. The mean percentage closure was calculated by compared with time 0 .

Cell migration assay. Cell migration was performed in Transwell chambers (tissue culture treated, $6.5 \mathrm{~mm}$ diameter, $8 \mu \mathrm{m}$ pores, Corning Inc., Corning, NY, USA) as described elsewhere [14]. Briefly, $2 \times 10^{4}$ cells in serum-free DMEM were placed into the upper chambers of Transwell inserts set within wells with $8 \mu \mathrm{m}$ pore filters, and incubated at $37^{\circ} \mathrm{C}$ for $12 \mathrm{~h}$. Cells on the upper surface of the chambers were then removed with cotton swabs. The migrated cells on the lower membrane surface were fixed in methanol and stained with $0.1 \%$ Giemsa stain. Eight microscope fields $(\times 200)$ from each Transwell chamber were randomly selected, and cells adhering to the undersurface of the filter were imaged and counted using an Olympus BX41 microscope equipped with a digital camera (Olympus, Tokyo, Japan).

Gelatine zymography assay. The assay was conducted according to published protocol [15]. Protein concentrations in the conditioned media were determined using the bicinchoninic acid method (BCA kit) (Pierce, Rockford, IL, USA). The conditioned media were mixed with an equal volume of $4 \times$ sample buffer $(200 \mathrm{mM}$ Tris- $\mathrm{HCl}, 8 \%$ SDS, $0.4 \%$ bromophenol blue, $40 \%$ glycerol) and electrophoresed on $6 \%$ SDS polyacrylamide gels containing $1 \mathrm{mg} / \mathrm{ml}$ of gelatine (Bio Basic Inc., Markham, Ontario, Canada). The gels were then washed twice for $30 \mathrm{~min}$ in $2.5 \%$ Triton $\mathrm{X}-100$ at room temperature, and incubated for $48 \mathrm{~h}$ at $37^{\circ} \mathrm{C}$ in incubation buffer (50 mM Tris- $\mathrm{HCl}$ (pH 7.5), $5 \mathrm{mM} \mathrm{CaCl} 2,150 \mathrm{mM} \mathrm{NaCl}, 1 \mu \mathrm{M}$ $\mathrm{ZnCl} 2$, and $0.2 \%$ Brij35). Gels were then stained for $1 \mathrm{~h}$ with $0.25 \%$ (w/v) Coomassie brilliant blue R-250, and de-stained in de-staining buffer (10\% acetic acid and 50\% methanol).

Confocal microscopy and ruffling assay. The assay was conducted according to published protocol [10]. The cells were seeded on glass coverslips in 6-well plates and pretreated with U73122, followed by EGF treatment. The treated cells were rinsed with PBS once and fixed in $4 \%$ paraformaldehyde for $10 \mathrm{~min}$, and then washed three times with PBS. After 5 min permeation with $0.5 \%$ Triton X-100, the cells were incubated with Rhodamine-conjugated Phalloidin (Cytoskeleton Inc., Denver, CO, USA) for $30 \mathrm{~min}$ at room temperature, before being stained with DAPI for $30 \mathrm{sec}$. Finally, the cells were observed and photographed using confocal microscopy.

Western blot analysis. The protein extracts were subjected to SDS-PAGE (8-10\%) and transferred to nitrocellulose membrane for Western blot analysis [16]. The membrane was incubated at $4^{\circ} \mathrm{C}$ overnight with various antibodies as required, which was followed by the addition of the corresponding secondary antibody at room temperature for 1 to $2 \mathrm{~h}$. An ECL kit was used to detect antibody reactivity (Pierce).

Immunohistochemical staining and analysis. The immunohistochemistry was performed as described previously [17, 18]. The tissue microarray was deparaffinised in xylene and rehydrated in graded alcohols and distilled water. Following antigen retrieval using the Citrate Buffer method - which can enhance the staining intensity of the antibodies by unmasking the antigens and epitopes in formalin-fixed and paraffin-embedded tissue sections - endogenous peroxidase activity was blocked with $3 \%$ hydrogen peroxide in methanol for $10 \mathrm{~min}$ at room temperature. This was followed by rehydration in PBS and incubation with $10 \%$ goat serum for $10 \mathrm{~min}$ to bind nonspecific antigens. As described in the manufacturer's instruction (MAIXIN.BIO, Fuzhou, China), the sections were incubated overnight at $4^{\circ} \mathrm{C}$ with PLC $\gamma 1$ (1:100 dilutions) primary antibody and subsequently with secondary antibody (1:400) for $60 \mathrm{~min}$ at room temperature. Diaminobenzidine (DAB) was used to visualize the immunohistochemical reaction, before counterstaining with haematoxylin. In the control sections, the specific primary antibody was omitted or replaced with non-immune serum or isotype-matched immunoglobulins. Photomicrographs were taken using an Olympus BX41 microscope equipped with a digital camera.

A semiquantitative scoring system based on staining intensity and the distribution of positive cells was used to evaluate PLC $\gamma 1$ expression $[18,19]$. The intensity of PLC $\gamma 1$ staining ranged from 0 to $3-$ i.e., negative $(-)$, weak $(+)$, moderate $(++)$, and strong $(+++)$, and was performed using Image-Pro Plus 6.0 Software. Two pathologists were consulted for agreement and the scores were quantified by three independent observers using the criteria for statistical analysis detailed in Figure 1C.

Statistical analysis. The differences between the groups were examined for statistical significance using the $\chi^{2}$ test, Student's $t$-test, and one-way ANOVA using SPSS software. A value of $p<0.05$ was considered significant. 
A

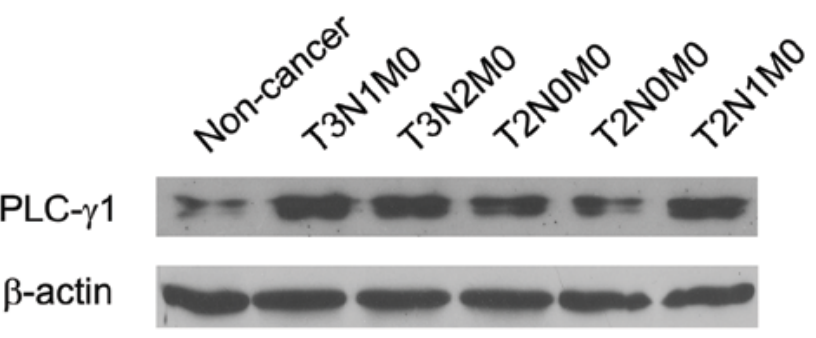

B

Non-cancer

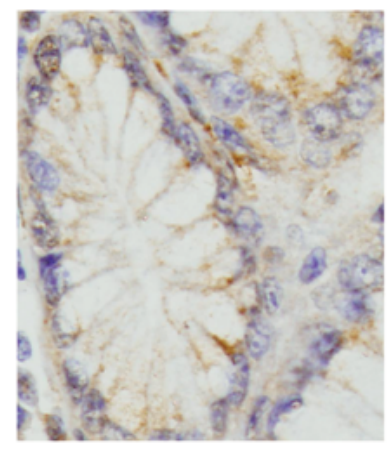

T3NOMO

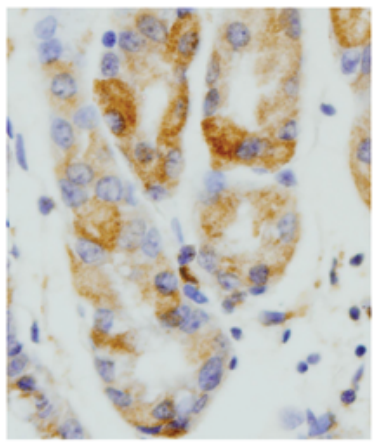

T3N2MO

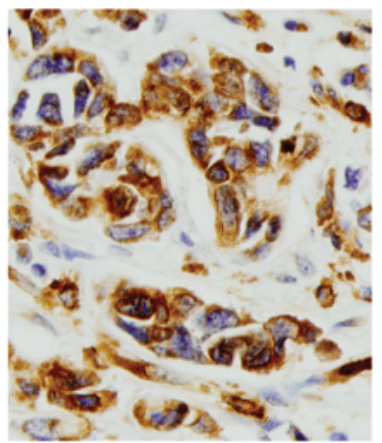

C

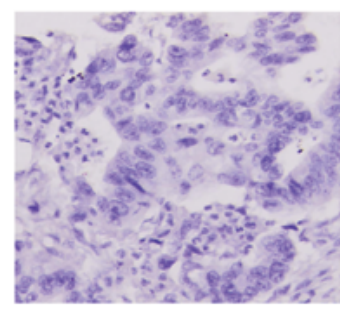

$-$

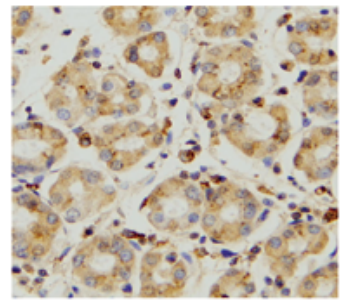

$++$

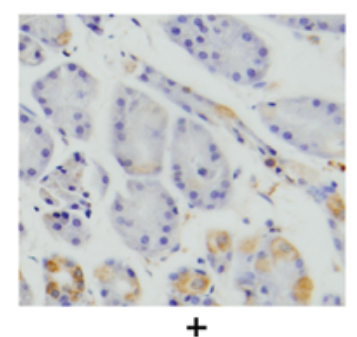

$+$

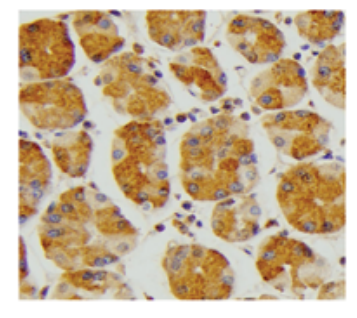

$+++$

Figure 1. PLC $\gamma 1$ is highly expressed in gastric adenocarcinoma with regional lymph node metastasis. A. PLC $\gamma 1$ expression in fresh human gastric adenocarcinoma and normal gastric mucosa tissues was detected using Western blot analysis with anti-PLC $\gamma 1$ and $\beta$-actin antibodies; B. PLC $\gamma 1$ expression in gastric adenocarcinoma and normal gastric mucosa tissues was detected in the tissue assay with immunohistochemical staining as described in the Material and methods section (magnification $\times 400$ ); C. Criteria for statistical analysis of PLC $\gamma 1$ expression in gastric adenocarcinoma tissues. PLC $\gamma 1$ expression in gastric adenocarcinoma tissues was detected in the tissue assay with immunohistochemical staining, as described in the Material and methods section (magnification $\times 200$ )

\section{Results}

\section{PLC $\gamma 1$ is highly expressed in human gastric adenocarcinoma}

Fresh human normal gastric mucosa tissue and gastric adenocarcinoma tissue were used for Western blot analysis of PLC $\gamma 1$. The results showed that PLC $\gamma 1$ expression was higher in human gastric adenocarcinoma than in normal gastric mucosa, while homogenously weak expression of PLC $\gamma 1$ was observed in normal gastric mucosa tissues (Figure 1A). Simultaneously, PLC $\gamma 1$ expression was higher in gastric ade- 
Table 1. Clinical pathological characteristics of PLC $\gamma 1$ in gastric adenocarcinoma

\begin{tabular}{|l|c|c|c|c|c|c|}
\hline & & \multicolumn{5}{|c|}{ PLC $\gamma \mathbf{1}$} \\
\cline { 2 - 7 } & Cases & - & + & $++/+++$ & $X^{2}$ & $P$ \\
\hline Tumor stage & & & & & & \\
I & 9 & 1 & 2 & 6 & 14.86 & $<0.01$ \\
II & 28 & 4 & 11 & 13 & & \\
III & 8 & 1 & 5 & 2 & & \\
\hline Metastasis & 28 & 4 & & & & \\
Non-metastasis & 17 & 2 & 3 & 9 & 6.813 & $<0.05$ \\
Regional lymph node metastasis & & & 12 & & \\
\hline
\end{tabular}

nocarcinoma tissues with lymph node metastasis (N1, N2) than in gastric adenocarcinoma tissues lacking lymph node metastasis (N0) (Figure 1A).

Subsequently, PLC $\gamma 1$ expression was assessed immunohistochemically on a tissue microarray. The majority $(86 \%)$ of gastric adenocarcinoma specimens (T2, T3, T4) showed a specific immune reactivity to PLC $\gamma 1$ (Figure 1B and 1C, Table 1, $\mathrm{p}<0.01$ ). PLC $\gamma 1$ expression was significantly higher in gastric adenocarcinoma specimens with regional lymph node metastasis $(\mathrm{N} 1, \mathrm{~N} 2)$ than in non-metastatic specimens (N0) (Figure $1 \mathrm{C}$, Table $1, \mathrm{p}<0.05$ ). These data indicate that PLC $\gamma 1$ expression is higher in gastric adenocarcinoma tissues than in normal gastric mucosa, and, specifically, in gastric adenocarcinoma tissues with regional lymph node metastasis.

\section{The migration of gastric adenocarcinoma cells partly depends on PLC $\gamma 1$ activation}

To investigate the role of PLC $\gamma 1$ in the migration of gastric adenocarcinoma cells, cells of the cultured gastric adenocarcinoma line BGC-823 were pretreated using PLC $\gamma 1$ inhibitor U73122 $(5 \mu \mathrm{mol} / \mathrm{L})$ for the indicated time in the presence or absence of EGF $(20 \mathrm{ng} / \mathrm{ml})[20,21]$. This was followed by a wound healing assay and Western blot analysis. The result of the wound healing assay showed that the addition of U73122 significantly suppressed the mean percentage closure of BGC-823 cells in the presence or absence of EGF treatment, compared with the untreated group, while the inhibitory effect of U73122 on the mean percentage closure of BGC-823 cells increased in a time-dependent manner (Figure 2A).

Simultaneously, Western blot analysis showed that the addition of PLC $\gamma 1$ inhibitor U73122 reduced the phosphorylation level of PLC $\gamma 1$ at the Y783 site (p-PLC $\gamma 1$ (Y783)), which is essential for PLC $\gamma 1$ activation [3], with a decrease in the expression level of MMP9 and MMP2 in the presence or absence of EGF treatment (Figure 2B).
Since several lines of evidence indicate that the PLC $\gamma 1$ substrate PtdIns(4,5)P2 and the enzyme itself were involved in cytoskeletal rearrangement through the modulation of actin-binding proteins [22, 23], we tested the effects of U73122 on actin cytoskeleton using confocal microscopy and a ruffling assay after rhodamine-conjugated phalloidin staining. The addition of U73122 led to a marked decrease in the number of membrane ruffles of BGC-823 cells in the presence or absence of EGF treatment (Figure $2 \mathrm{C}$ ), with the reduction of actin cytoskeleton reorganization in cytoplasm (Figure 2D, indicated as white arrows). Taken together, these data suggest that the migration of BGC-823 cells partly depends on PLC $\gamma 1$ activation.

\section{Akt is involved in PLC $\gamma 1$-dependent migration of gastric adenocarcinoma cells}

To confirm the role of Akt in PLC $\gamma 1$-dependent migration of gastric adenocarcinoma cells, BGC-823 cells were treated with an Akt inhibitor Triciribine (TCN) for the indicated time, and PLC $\gamma 1$ expression and activation were assessed by Western blot analysis.

The addition of TCN $(10 \mu \mathrm{mol} / \mathrm{L})$ significantly suppressed PLC $\gamma 1$ and Akt activation, with a slight downregulation of total PLC $\gamma 1$ expression (Figure 3A). Similar results were observed in BGC-823 cells with the transfection of the myc-Akt vector. Overexpression of Akt upregulated the expression level of PLC $\gamma 1$ and its activation (p-PLC $\gamma 1$ (Y783)), while the expression of p-PLC $\gamma 1$ (S1248) was also upregulated, albeit to a lower extent (Figure 3B). Simultaneously, overexpression of Akt upregulated the expression level of MMP2/9 (Figure 3B).

Interestingly, the phosphorylation level of ERK (p-ERK) also was upregulated in the BGC-823 cells transfected with overexpression of the Akt vector (Figure 3B). EGF treatment enhanced the effect of Akt on p-PLC $\gamma 1$ (Y783), p-PLC $\gamma 1$ (S1248), p-ERK, and MMP2/9 expression. Furthermore, only HA-PLC $\gamma 1$ 

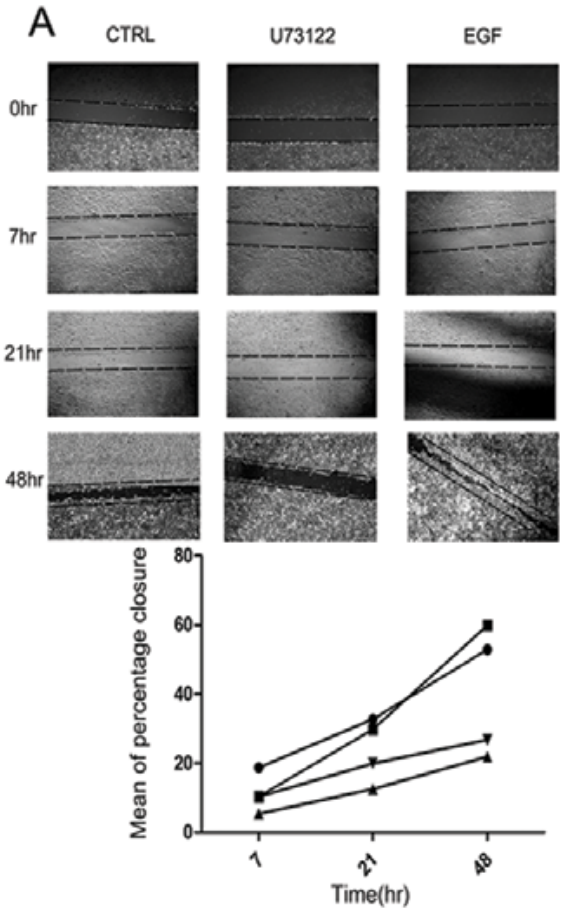

C

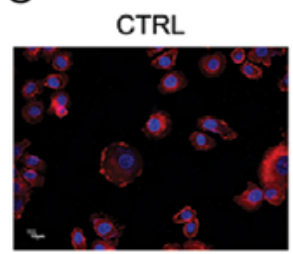

EGF
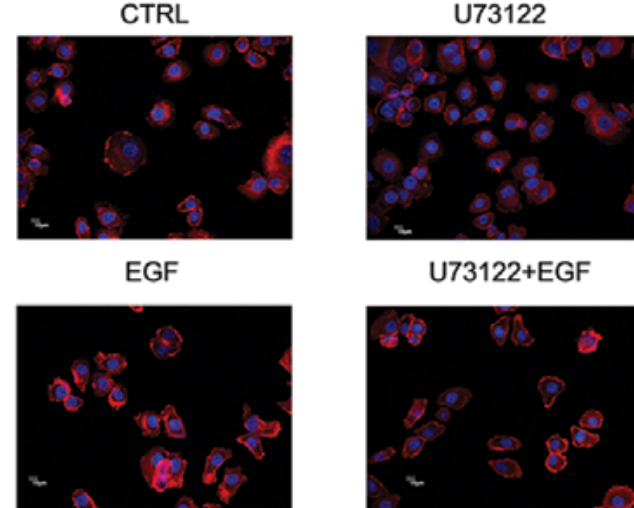

U73122+EGF
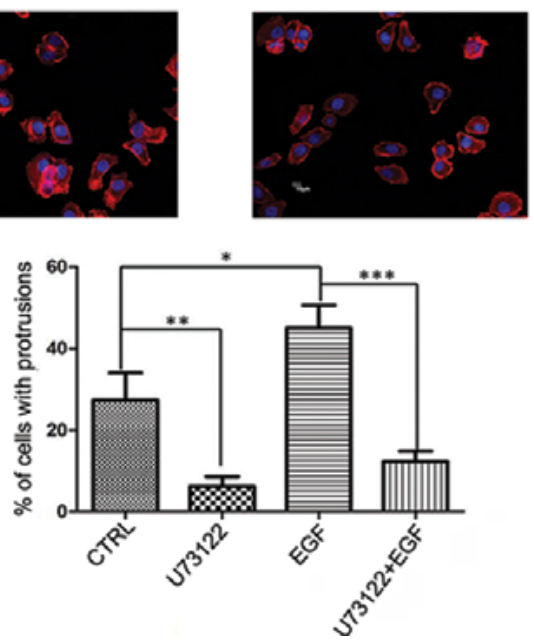

B
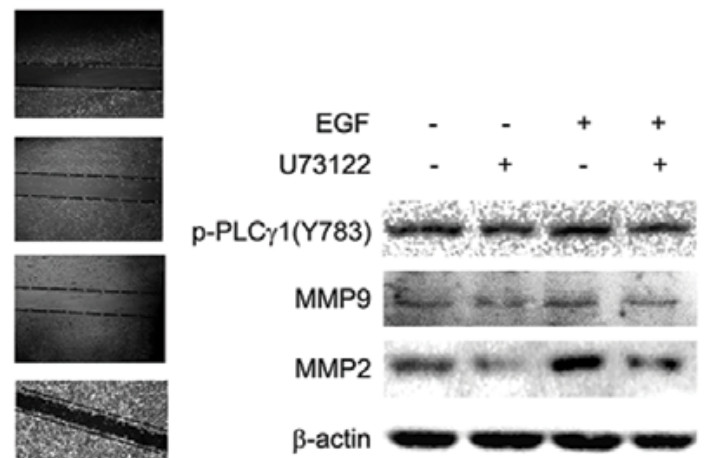

D

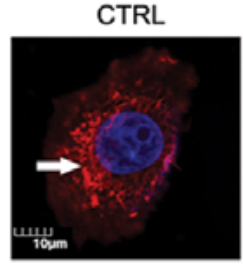

EGF
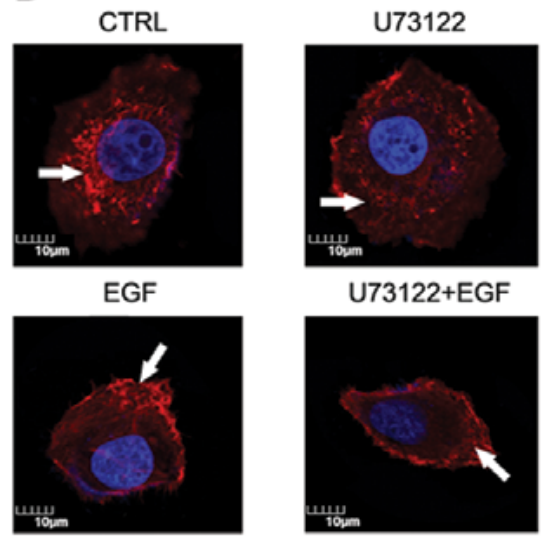

$\mathrm{U} 73122+\mathrm{EGF}$

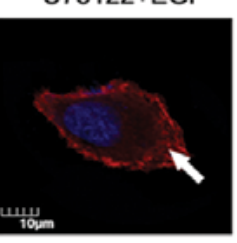

Figure 2. The migration of BGC-823 cells partly depends on PLC $\gamma 1$ activation. A. Cells were treated with or without U73122 $(5 \mu \mathrm{mol} / \mathrm{L})$ for the indicated time in the presence or absence of EGF $(20 \mathrm{ng} / \mathrm{ml})$; this was followed by wound healing assays, as described in the Material and methods section (magnification $\times 40$ ); B. Cells were pretreated with U73122 $(5 \mu \mathrm{mol} / \mathrm{L})$ for $2 \mathrm{~h}$, and then treated with EGF $(20 \mathrm{ng} / \mathrm{ml})$ for $0.5 \mathrm{~h}$. Protein expressions of p-PLC $\gamma 1$ (Y783), MMP2, MMP9, and $\beta$-actin were detected using Western blot analysis with the corresponding antibodies; C. Cells were pretreated with $\mathrm{U} 73122(5 \mu \mathrm{mol} / \mathrm{L})$ for $2 \mathrm{~h}$ and then treated with EGF $(20 \mathrm{ng} / \mathrm{ml})$ for $1 \mathrm{~h}$. The formation of membrane ruffles was then detected using confocal microscopy and ruffling assay following Rhodamine-conjugated Phalloidin staining, as described in the Material and methods section (magnification $\times 400,{ }^{*} \mathrm{p}<0.05,{ }^{* *} \mathrm{p}<0.01$, and ${ }^{* * *} \mathrm{p}<0.001$ ); D. Cells were treated as in Figure 2C, and the effect of U73122 on actin cytoskeleton was observed using confocal microscopy following Rhodamine-conjugated Phalloidin staining. The white arrows indicate the actin aggregation (magnification $\times 800$ ). The data are representative of three or five independent experiments, each yielding similar results 
A

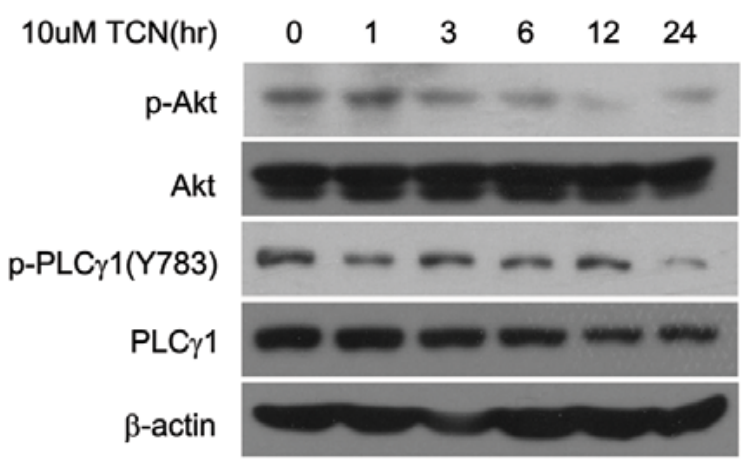

B

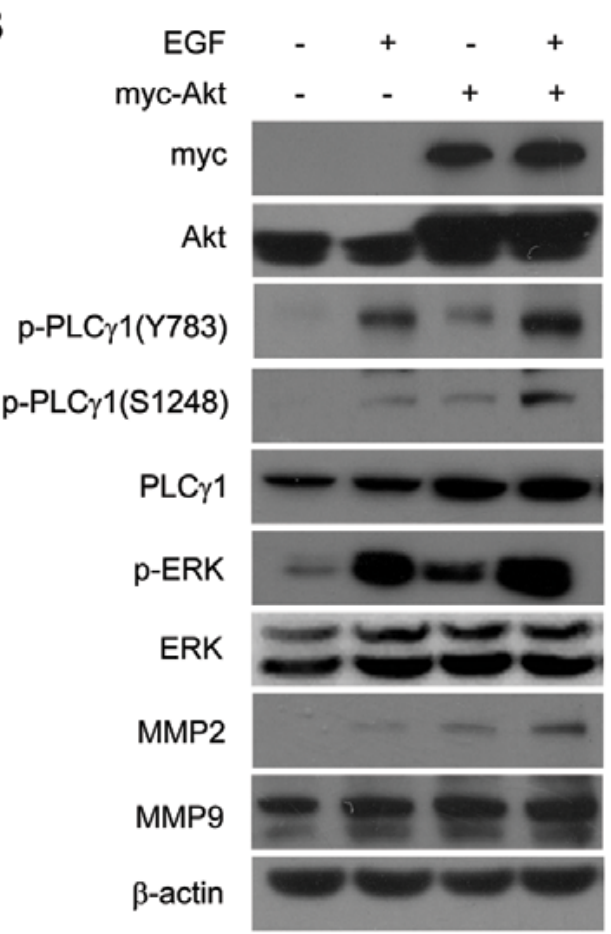

D

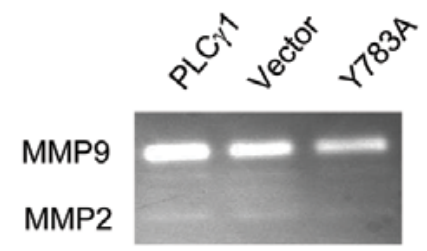

E

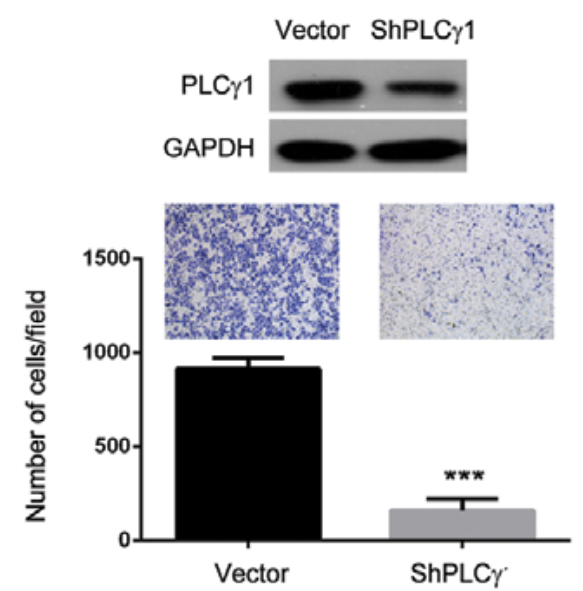

Figure 3. PLC $\gamma 1$-dependent cell migration is related to Akt expression in BGC-823 cells. A. Cells were treated with TCN $(10 \mu \mathrm{mol} / \mathrm{L})$ for the indicated time; the protein expression of PLC $\gamma 1$, p-PLC $\gamma 1$ (Y783), Akt, p-Akt (S473), and $\beta$-actin was then detected using Western blot analysis with the corresponding antibodies; B. The myc-Akt vector was transfected into cells and followed by treatment with EGF $(20 \mathrm{ng} / \mathrm{ml})$ for $0.5 \mathrm{~h}$; the protein expression of PLC $\gamma 1$, p-PLC $\gamma 1$ (Y783), p-PLC $\gamma 1$ (S1248), ERK, p-ERK1/2 (T202/Y204), MMP2, MMP3, Akt, myc, and $\beta$-actin was then detected using Western blot analysis with the corresponding antibodies; C. HA-PLC $\gamma 1$, HA-PLC $\gamma 1$ Y783A, and HA-PLC $\gamma 1$ S1248A vectors were transfected into cells, and the protein expression of PLC $\gamma 1$, p-PLC $\gamma 1$ (Y783), p-PLC $\gamma 1$ (S1248), ERK, p-ERK1/2 (T202/Y204), HA, and $\beta$-actin was detected using Western blot analysis with the corresponding antibodies; D. Cells were transfected with HA-PLC $\gamma 1$ and HA-PLC $\gamma 1$ Y783A vectors; this was followed by the gelatine zymography assay described in the Material and methods section; E. Cells were transfected with ShPLC $\gamma 1$ vector; this was followed by the Transwell assay described in the Material and methods section $\left({ }^{* * *} \mathrm{p}<0.001\right)$. The data are representative of three or five independent experiments, each yielding similar results 
Y783A significantly reduced the expression level of p-ERK in BGC-823 cells transfected with HA-PLC $\gamma 1$, HA-PLC $\gamma 1$ Y783A, and HA-PLC $\gamma 1$ S1248A vectors, showing that PLC $\gamma 1$ activation (p-PLC $\gamma 1$ (Y783)) was required for ERK phosphorylation in BGC-823 cells (Figure 3C).

At the same time, HA-PLC $\gamma 1$ Y783A significantly reduced the expression of MMP2/9, as seen using gelatine zymography assay (Figure 3D), and the results of the Transwell assay demonstrated that the transfection of ShPLC $\gamma 1$ vector into the BGC-823 cells reduced the number of cell migrations (Figure $\left.3 \mathrm{E},{ }^{* * *} \mathrm{p}<0.001\right)$. Therefore, the data indicate the involvement of Akt in PLC $\gamma 1$-dependent migration of gastric adenocarcinoma cells, in which Akt triggers the PLC $\gamma /$ ERK/MMP pathway.

\section{Discussion}

In this study, we demonstrated that PLC $\gamma 1$ expression was related to the dissemination of gastric adenocarcinoma. Both the migration of cells and reorganization of the actin cytoskeleton partly depended on PLC $\gamma 1$ activation in the BGC-823 gastric adenocarcinoma cell line. This activation was mediated through Akt, an upstream of PLC $\gamma 1$ that triggered PLC $\gamma 1 /$ ERK/MMP pathway in the BGC-823 cells. Thus, PLC $\gamma 1$ activities may play an important role in the dissemination of gastric adenocarcinoma cells.

As one of switches that triggers cell migration, PLC $\gamma 1$ appears to be at the convergence point of various signalling pathways leading to cell cytoskeleton rearrangement and motility [24]. In line with previous studies of other tumors [8-10,25], the data from the tissue microarray and human tumor specimens demonstrate that PLC $\gamma 1$ expression is related to the dissemination of gastric adenocarcinoma cells. Furthermore, the disruption of PLC $\gamma 1$ led to the attenuation of cell migration and a decrease in both the number of cell membrane ruffles and the incidence of actin reorganization in BGC-823 cells. Thus, the migration of gastric adenocarcinoma cells partly depends on PLC $\gamma 1$ activation, and blocking PLC $\gamma 1$ could be a potential therapeutic approach to prevent gastric adenocarcinoma metastasis.

Although several lines of evidence indicated that the interaction between Akt and PLC $\gamma$ is involved in cell growth and migration [11, 12], some studies argued against the regulatory mechanisms of the two signalling pathways in this process, whether upstream, downstream, or with each other. Browaeys-Poly et al. reported that Akt was known to phosphorylate its substrate, PLC $\gamma$, and then to regulate the G2/M transition triggered by FGF receptors in MDA-MB-231 breast cancer cells [12]. However, Li et al. observed that Akt was phosphorylated to stimulate cell proliferation through the activated PLC $\gamma / \mathrm{PKC} \gamma / \mathrm{Src} / \mathrm{PI} 3 \mathrm{~K}$ pathway in EGF-stimulated rat and human conjunctival goblet cells [26]. Our data, indicating that Akt phosphorylates PLC $\gamma 1$ at the Y783 site and promotes cell migration, is consistent with Browaeys-Poly's study [12]. The different regulatory mechanisms of the two signalling pathways might be dependent on cellular context and type. Previous studies found that the PLC $\gamma 1$ activation was also regulated by phosphorylation at an additional serine/threonine site, even though PLC $\gamma 1$ was activated by tyrosine phosphorylation [27, 28]. Likewise, Akt simultaneously phosphorylates PLC $\gamma 1$ at the Y783 and S1248 sites in BGC-823 cells. We thus suggest that Akt, as an upstream, interacts with PLC $\gamma 1$, activates PLC $\gamma 1$ at the $\mathrm{Y} 783$ and S1248 sites in BGC-823 cells, and is involved in PLC $\gamma 1$-dependent migration. Further investigation into the role of p-PLC $\gamma 1$ (S1248) phosphorylated by Akt in gastric adenocarcinoma cell migration is underway.

In addition, ERK1/2, as a downstream effect of PLC $\gamma 1$, has been described as regulating MMP expression [29, 30]. The association of Ras-GRF1/2 with PLC $\gamma 1$ enables PLC $\gamma 1$ to be recruited to focal adhesions and is required for Ras signalling, ERK activation, and MMP-3 release downstream of IL-1 stimulation [29]. It was shown that PLC $\gamma 1$ could activate ERK1/2 through the PLC $\gamma 1$-PKC $\gamma$-B-Raf pathway in VEGF-treated endothelial cells [30]. Consistent with previous studies, our data also indicate that PLC $\gamma 1$ activation is required for the phosphorylation of ERK. Combined with our result that the phosphorylation of ERK increases with the transfection of the myc-Akt vector in the presence or absence of EGF treatment, we suggest that Akt could mediate PLC $\gamma 1$ activation, which would trigger the PLC $\gamma 1 /$ ERK/MMP pathway to modulate the migration of BGC- 823 cells.

In summary, in this study we have shown that PLC $\gamma 1$ activation is partly required for the migration of gastric adenocarcinoma cells. Moreover, this Akt-mediated activation triggers the PLC $\gamma 1 /$ ERK/MMP pathway in BGC-823 cells. Thus, the activation of PLC $\gamma 1$ and its interaction with Akt underline PLC $\gamma 1$ as a potential therapeutic target in gastric adenocarcinoma.

\section{Acknowledgments}

We would like to thank Dr. Qiao Wu (Xiamen University, Xiamen, Fujian, China) for the generous supply of the myc-Akt vector. This study was supported by the National Natural Science Foundation of China (No. 81072015, 81371952), the Medical Innovation 
Foundation of Fujian, China (No. 2011-CXB-36), and the Natural Science Foundation of Xiamen, China (No. 3502Z20124046, 3502Z2013016).

\section{References}

1. Miao RL, Wu AW. Towards personalized perioperative treatment for advanced gastric cancer. World J Gastroenterol. 2014;20:11 586-11 594.

2. Parkin DM, Bray F, Ferlay J, Pisani P. Global cancer statistics, 2002. CA Cancer J Clin. 2005;55:74-108.

3. Tongyoo A. Targeted therapy: novel agents against cancer. J Med Assoc Thai. 2010;93(Suppl 7):S311-S323.

4. Polivka J Jr, Janku F. Molecular targets for cancer therapy in the PI3K/AKT/mTOR pathway. Pharmacol Ther. 2014;142:164-175.

5. Lattanzio R, Piantelli M, Falasca M. Role of phospholipase C in cell invasion and metastasis. Adv Biol Regul. 2013;53:309-318.

6. Rhee SG. Regulation of phosphoinositide-specific phospholipase C. Annu Rev Biochem. 2001;70:281-312.

7. Wells A, Grandis JR. Phospholipase C- $\gamma 1$ in tumor progression. Clin Exp Metastasis. 2003;20:285-290.

8. Yamaguchi H, Condeelis J. Regulation of the actin cytoskeleton in cancer cell migration and invasion. Biochim Biophys Acta. 2007;1773:642-652.

9. Shepard CR, Kassis J, Whaley DL et al. PLC gamma contributes to metastasis of in situ-occurring mammary and prostate tumors. Oncogene. 2007;26:3020-3026.

10. Sala G, Dituri F, Raimondi C et al. Phospholipase C-gamma1 is required for metastasis development and progression. Cancer Res. 2008;68:10 187-10 196.

11. Tomas NM, Masur K, Piecha JC et al. Akt and phospholipase $\mathrm{C} \gamma$ are involved in the regulation of growth and migration of MDA-MB-468 breast cancer and SW480 colon cancer cells when cultured with diabetogenic levels of glucose and insulin. BMC Res Notes. 2012;5:214.

12. Browaeys-Poly E, Perdereau D, Lescuyer A et al. Akt interaction with PLC(gamma) regulates the $\mathrm{G}(2) / \mathrm{M}$ transition triggered by FGF receptors from MDA-MB-231 breast cancer cells. Anticancer Res. 2009;29:4965-4969.

13. Liang CC, Park AY, Guan JL. In vitro scratch assay: a convenient and inexpensive method for analysis of cell migration in vitro. Nat Protoc. 2007;2:329-333.

14. Beloueche-Babari M, Peak JC, Jackson LE et al. Changes in choline metabolism as potential biomarkers of phospholipase $\mathrm{C}$ (gamma) 1 inhibition in human prostate cancer cells. Mol Cancer Ther. 2009;8:1305-1311.

15. Ruan M, Zhang Z, Li S et al. Activation of Toll-Like Receptor-9 promotes cellular migration via up-regulating MMP-2 expression in oral squamous cell carcinoma. PLoS One. 2014;9:e92748.

16. Zhang B, Xia C. 12-O-tetradecanoylphorbol-1,3-acetate-induced degradation of protein kinase $\mathrm{B}$ via ubiquitin pro- teasomal pathway depends on its Ser473 phosphorylation in gastric cancer cells. Folia Histochem Cytobiol. 2013;51: 11-17.

17. Chen Q, Zhang B, Yi T et al. Increased apoptosis in human knee osteoarthritis cartilage related to the expression of protein kinase $\mathrm{B}$ and protein kinase $\mathrm{C} \alpha$ in chondrocytes. Folia Histochem Cytobiol. 2012;50:137-143.

18. Mao Y, Zhang N, Xu J et al. Significance of heterogeneous Twist 2 expression in human breast cancers. PLoS One. 2012;7:e48 178.

19. Chen J, Wei D, Zhao Y et al. Overexpression of EFEMP1 correlates with tumor progression and poor prognosis in human ovarian carcinoma. PLoS One. 2013;8:e78 783.

20. Yao TW, Kim WS, Yu DM et al. A novel role of dipeptidyl peptidase 9 in epidermal growth factor signaling. Mol Cancer Res. 2011;9:948-959.

21. Cho M, Kabir SM, Dong Y et al. Aspirin blocks EGF-stimulated cell viability in a COX-1 dependent manner in ovarian cancer cells. J Cancer. 2013;4:671-678.

22. Halstead JR, Jalink K, Divecha N. An emerging role for PtdIns(4, 5)P2-mediated signalling in human disease. Trends Pharmacol Sci. 2005;26:654-660.

23. van Rheenen J, Song X, van Roosmalen W et al. EGF-induced PIP2 hydrolysis releases and activates cofilin locally in carcinoma cells. J Cell Biol. 2007;179:1247-1259.

24. Jones NP, Peak J, Brader S et al. PLCg1 is essential for early events in integrin signaling required for cell motility. $J$ Cell Sci. 2005;118:2695-2706.

25. Lattanzi R, Marchisio M, La Sorda R et al. Overexpression of activated phospholipase $\mathrm{C} \gamma 1$ is a risk factor for distant metastases in T1-T2, N0 breast cancer patients undergoing adjuvant chemotherapy. Int J Cancer. 2013;132:1022-1031 .

26. Li D, Shatos MA, Hodges RR et al. Role of PKC $\alpha$ activation of Src, PI-3K/AKT, and ERK in EGF-stimulated proliferation of rat and human conjunctival goblet cells. Invest Ophthalmol Vis Sci. 2013;54:5661-5674.

27. Choi JH, Ryu SH, Suh PG. On/off-regulation of phospholipase C-gamma 1-mediated signal transduction. Adv Enzyme Regul. 2007;47:104-116.

28. Bae SS, Choi JH, Oh YS et al. Regulation of phospholipase $\mathrm{C}$-gamma1 by protein kinase A-dependent phosphorylation. Adv Enzyme Regul. 2002;42:195-211.

29. Wang Q, Siminovitch KA, Downey GP et al. Ras-guanine-nucleotide-releasing factors 1 and 2 interact with PLC $\gamma$ at focal adhesions to enable IL-1-induced $\mathrm{Ca}(2+)$ signalling, ERK activation and MMP-3 expression. Biochem J. 2013;449:771-782.

30. Andrikopoulos P, Baba A, Matsuda $\mathrm{T}$ et al. Ca2+ influx through reverse mode $\mathrm{Na}+/ \mathrm{Ca} 2+$ exchange is critical for vascular endothelial growth factor-mediated extracellular signal-regulated kinase (ERK) 1/2 activation and angiogenic functions of human endothelial cells. J Biol Chem. 2011;286: 37 919-37931. 